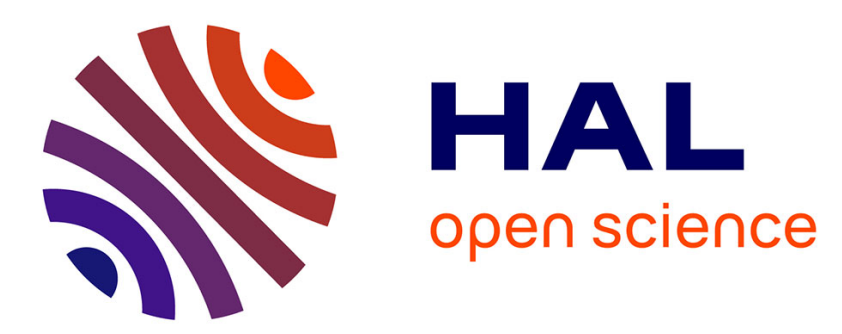

\title{
An improvement of the uncertainty quantification in computational structural dynamics with nonlinear geometrical effects \\ Evangéline Capiez-Lernout, Christian Soize
}

\section{- To cite this version:}

Evangéline Capiez-Lernout, Christian Soize. An improvement of the uncertainty quantification in computational structural dynamics with nonlinear geometrical effects. International Journal for Uncertainty Quantification, 2017, 7 (1), pp.83-98. 10.1615/Int.J.UncertaintyQuantification.2016019141 . hal-01465405

\section{HAL Id: hal-01465405 \\ https://hal.science/hal-01465405}

Submitted on 11 Nov 2017

HAL is a multi-disciplinary open access archive for the deposit and dissemination of scientific research documents, whether they are published or not. The documents may come from teaching and research institutions in France or abroad, or from public or private research centers.
L'archive ouverte pluridisciplinaire HAL, est destinée au dépôt et à la diffusion de documents scientifiques de niveau recherche, publiés ou non, émanant des établissements d'enseignement et de recherche français ou étrangers, des laboratoires publics ou privés. 


\title{
AN IMPROVEMENT OF THE UNCERTAINTY QUAN- TIFICATION IN COMPUTATIONAL STRUCTURAL DYNAMICS WITH NONLINEAR GEOMETRICAL EF- FECTS.
}

\author{
Evangeline Capiez-Lernout* \& Christian Soize
}

Laboratoire Modelisation et Simulation Multi-Echelle, MSME UMR 8208 CNRS, Universite Paris-Est, Marne-La-Vallee, France

Original Manuscript Submitted: 12/19/2016; Final Draft Received: 12/19/2016

\begin{abstract}
In this work, we present an improvement of a computational methodology for the uncertainty quantification of structures in presence of geometric nonlinearities. The implementation of random uncertainties is carried out through the nonparametric probabilistic framework from a nonlinear reduced-order model. With such usual modeling, it is difficult to analyze the influence of uncertainties on the nonlinear part of the operators with respect to its linear counterpart. In order to address this problem, an approach is proposed to take into account uncertainties for both the linear and the nonlinear operators. The methodology is then validated in the context of the nonlinear post-buckling of a cylindrical shell and in the context of a nonlinear mistuned industrial integrated bladed-disk.
\end{abstract}

KEY WORDS: Geometric nonlinearities, Uncertainty quantification, Nonlinear reduced-order model, Nonlinear structural dynamics

\section{INTRODUCTION}

This work proposes an improvement of the uncertainty quantification in computational structural dynamics with nonlinear geometrical effects, based on the nonparametric probabilistic approach. With respect to all the previous published works, the novelties presented in this paper concern (1) an improvement of the numerical implementation of the stochastic modeling by introducing a second statistical reduction related to the nonlinear stochastic operators, (2) a validation on two industrial applications, and (3) an analysis concerning the existence of several uncertain branches in the stochastic post-buckling behavior of thin cylindrical shells.

In this paper, we focus on the probabilistic aspects for modeling uncertainties when dealing with structures which undergo nonlinear geometrical effects induced by the large strains and large displacements. Hereinafter, we begin this introduction with a brief history concerning structural dynamical analyses for which nonlinear phenomena combined with the presence of uncertainties are taken into account. Thanks to the improved performance of multi-core processors adapted to the parallelization and to the distribution of the numerical computation, associated with technological advances such as the elaboration of new multi-functional materials and 3D-printing technology for manufacturing optimized structures with complex shapes, there are many industrial applications, which require such modeling in order to obtain robust predictions with respect to uncertainties in the computational models.

One essential aspect is to pay a particular attention to the nonlinear effects that can occur. Many research efforts have been made in the field of modeling local nonlinearities [1-3] such as nonlinear contact interfaces, but also on global nonlinearities in a civil engineering context [4] as well as in a noise and vibration isolation context [5], in the turbomachinery context [6-8] or for thin or slender structures

*Correspond to: Evangeline Capiez-Lernout, E-mail: evangeline.capiez-lernout@u-pem.fr 
[9-15]. More sophisticated developments have been made in a finite element context by developing nonlinear reduced-order models [16-24].

Moreover, in many industrial applications, for which the investigated structures present a high complexity level, deterministic nonlinear computational models are in general not sufficient to accurately predict their dynamical behavior. Uncertainties have then to be taken into account in the computational models by using probabilistic models as soon as the probability theory can be used. Recently, a computational methodology adapted to the uncertainty quantification of structures in presence of geometric nonlinearities has been proposed and validated in the context of structural dynamics [20,25-27]. The implementation of random uncertainties is carried out through the nonparametric probabilistic framework from a nonlinear reduced-order model. It has been shown that such approach was adapted to the geometrical nonlinear case, by introducing a dedicated stiffness positive- definite operator self-containing the integrality of the information concerning the nonlinear geometric stiffness contributions. Nevertheless, its dimension increases with the square of the dimension of the nonlinear-reduced order model, requiring a large number of uncertain random germs. Consequently, establishing criteria for analyzing the influence of uncertainties on the nonlinear part of the operators with respect to its linear counterpart is difficult. The main idea for reducing the size of the random germ is to use a second reduction before implementing the model of uncertainties. It is then possible to coherently take into account uncertainties on both the linear operator and the nonlinear one.

The paper is organized as follows. In Section 2, after summarizing the main steps of the complete methodology, we present the implementation of the model uncertainties. After recalling the usual way to implement uncertainties on the linear or nonlinear operators in the context of the nonparametric probabilistic method, it is pointed out that both situations do not appear to be compatible since no meaningful comparison can be made. A reasonable and logical requirement consists in using the same random germ for both situations, with a feasibility constraint controlled by the size of the random germ. This is achieved by replacing the Cholesky factorization of the dedicated stiffness positive-definite operator by another factorization obtained from a usual eigenvalue problem for which only the contributions related to the largest eigenvalues are kept. In Section 3, the methodology is applied to two realistic numerical applications. The first one concerns the nonlinear static analysis of the post-buckling of an uncertain thin cylindrical shell and the second one deals with the nonlinear dynamical analysis of a mistuned industrial centrifugal compressor.

\section{CONSTRUCTION OF THE COMPUTATIONAL MODEL}

\subsection{Overview of the computational methodology}

Recently, a complete methodology has been proposed for the nonlinear dynamical or static analysis of uncertain structures taking into account the nonlinear geometrical effects induced by large displacements and large strains that can occur in the structure. The main steps of such methodology can be briefly summarized as follows.

i. Construction of the finite element model for the structure.

ii. Construction of a projection basis adapted to the problem analyzed. Such construction can be achieved by using, for instance, Proper Orthogonal Decomposition on the nonlinear static reference response of the structure (without any uncertainties) [28-31] or by using the elastic modes of the underlying mean linear dynamical computational model. The projection basis is represented by a matrix denoted by $[\Phi]$.

iii. Direct construction of the mean nonlinear reduced-order model by projection of the finite element operators on the constructed projection basis $[20,26]$. This means that the Galerkin method is applied to the boundary value problem yielding the continuous reduced-order model, and that 
the nonlinear operators related to this obtained reduced-order model are discretized by the finite element method.

iv. Construction of the external loads corresponding to (1) an incremental static load adapted to the nonlinear static context, (2) a time load for which its Fourier transform is constant in the chosen frequency band of excitation and allowing the nonlinear forced response to be computed in the time domain $[27,32]$.

v. Implementation of the uncertainties through the nonparametric probabilistic framework [33] in a nonlinear geometrical context.

vi. Computation of the response of the stochastic nonlinear differential equation in the time domain by using an adapted efficient algorithm based on the arc-length methods [26, 27]. The solution of this stochastic nonlinear reduced-order model is calculated using the Monte Carlo numerical simulation, using an implicit and unconditionally stable integration scheme (Newmark method with the averaging acceleration scheme) combined with either the fixed point method or the Crisfield arc-length based algorithm [27, 34], depending on the nonlinearity rate. A posterior nonlinear dynamical analysis is performed in the frequency domain by using Fast Fourier Transform.

All the details concerning the theoretical construction of such methodology and its applications in an industrial context can be found in $[20,27]$.

\subsection{Focus on the modeling of uncertainties}

From now on, this Section is focussed on item v. of Section 2.1, which concerns the numerical modeling of uncertainties from the nonparametric probabilistic methodology introduced in [35]. The main question concerns the capability of introducing uncertainties when nonlinear geometric effects occur depending on the fact that these nonlinearities can be considered either deterministic or uncertain.

\subsubsection{Stochastic nonlinear reduced order model}

In a general way, the stochastic nonlinear reduced-order model is described by the following stochastic nonlinear differential equation of order $M$, whose solution is described by the $\mathbb{R}^{M}$-valued random generalized coordinates vector $\mathbf{Q}$ :

$$
[\mathcal{M}] \ddot{\mathbf{Q}}+([\mathcal{D}]+[\mathcal{C}(\Omega)]) \dot{\mathbf{Q}}+\left(\left[\mathcal{K}^{(e)}\right]+\left[\mathcal{K}^{(c)}(\Omega)\right]+\left[\mathcal{K}^{(g)}\right]\right) \mathbf{Q}+\mathcal{F}^{\mathrm{NL}}(\mathbf{Q})=\mathcal{F},
$$

in which the reduced operators $[\mathcal{M}],\left[\mathcal{K}^{(g)}\right]$ and $\left[\mathcal{K}^{(e)}\right]$ are the random mass, geometrical stiffness, and elastic stiffness real matrices with values in the set of the positive-definite $(M \times M)$ matrices. The reduced operators $[\mathcal{C}(\Omega)]$ and $\left[\mathcal{K}^{(c)}(\Omega)\right]$ are related to the rotational motion of the structure with constant velocity $\Omega$. These operators represent the random gyroscopic coupling term with values in the set of the skew-symmetric $(M \times M)$ matrices and the random centrifugal stiffness term with values in the set of the negative-definite $(M \times M)$ matrices. In Eq. (1), the components of the nonlinear reduced internal force $\mathcal{F}^{\mathrm{NL}}(\mathbf{Q})$, resulting from the presence of geometric nonlinearities, is defined by

$$
\mathcal{F}_{\alpha}^{\mathrm{NL}}(\mathbf{Q})=\mathcal{K}_{\alpha \beta \gamma}^{(2)} Q_{\beta} Q_{\gamma}+\mathcal{K}_{\alpha \beta \gamma \delta}^{(3)} Q_{\beta} Q_{\gamma} Q_{\delta},
$$

in which the geometric nonlinearities are taken into account through the quadratic and the cubic stiffness contributions $\mathcal{K}_{\alpha \beta \gamma}^{(2)}$ and $\mathcal{K}_{\alpha \beta \gamma \delta}^{(3)}$. Note that the detailed expressions of these operators and of the external force $\mathcal{F}$ can be found in $[20,27]$. The random nonlinear displacement $\mathbf{U}$ is written as

$$
\mathbf{U}=[\Phi] \mathbf{Q} .
$$

The stochastic solver is briefly summarized in item vi. of Section 2.1. 


\subsubsection{Recalling a strategy for modeling uncertainties based on the nonparametric probabilistic method}

Model 1 (M1). The classical use of the nonparametric modeling of uncertainties would consist in introducing random matrices of the uncertain linear operators, keeping the nonlinear geometric terms deterministic. In that case, the random matrix $\left[\mathcal{K}^{(e)}\right]$ would be defined by

$$
\left[\mathcal{K}^{(e)}\right]=\left[L_{K^{(e)}}\right]^{\mathrm{T}}\left[\mathbf{G}_{K^{(e)}}\left(\delta_{K^{(e)}}\right)\right]\left[L_{K^{(e)}}\right],
$$

in which $\left[\mathcal{K}^{(e)}\right]$ would be the $(M \times M)$ upper triangular matrix issued from the Cholesky factorization of $\mathcal{E}\left\{\left[\mathcal{K}^{(e)}\right]\right\}=\left[\mathcal{K}^{(e)}\right]$ defined by the corresponding mean reduced deterministic model, and where $\left[\mathbf{G}_{K^{(e)}}\left(\delta_{K^{(e)}}\right)\right]$ would be a full random matrix with values in the set of all the positive-definite symmetric $(M \times M)$ matrices.

Model 2 (M2). In the approach proposed in [25] and implemented in [20, 26, 36], the uncertainties are globally introduced on all linear and nonlinear stiffness terms. This requires to explicitly construct the $(P \times P)$ real matrix $[\mathcal{K}]$ with

$$
P=M(M+1)
$$

defined by

$$
[\mathcal{K}]=\left[\begin{array}{cc}
{\left[\mathcal{K}^{(e)}\right]} & {\left[\widehat{\mathcal{K}}^{(2)}\right]} \\
{\left[\widehat{\mathcal{K}}^{(2)}\right]^{T}} & 2\left[\mathcal{K}^{(3)}\right]
\end{array}\right],
$$

in which the matrix blocks $\left[\widehat{\mathcal{K}}^{(2)}\right]$ and $\left[\mathcal{K}^{(3)}\right]$ are the $\left(M \times M^{2}\right)$ and the $\left(M^{2} \times M^{2}\right)$ real matrices resulting from the following reshaping operation

$$
\begin{gathered}
{\left[\widehat{\mathcal{K}}^{(2)}\right]_{\alpha J}=\widehat{\mathcal{K}}_{\alpha \beta \gamma}^{(2)}, J=(\beta-1) M+\gamma,} \\
{\left[\mathcal{K}^{(3)}\right]_{I J}=\mathcal{K}_{\alpha \beta \gamma \delta}^{(3)}, I=(\alpha-1) M+\beta, J=(\gamma-1) M+\delta .}
\end{gathered}
$$

In Eq.(7), the quadratic operator $\widehat{\mathcal{K}}^{(2)}$ is such that

$$
\mathcal{K}_{\alpha \beta \gamma}^{(2)}=\frac{1}{2}\left(\widehat{\mathcal{K}}_{\alpha \beta \gamma}^{(2)}+\widehat{\mathcal{K}}_{\beta \gamma \alpha}^{(2)}+\widehat{\mathcal{K}}_{\gamma \alpha \beta}^{(2)}\right)
$$

It has been shown in [25] that matrix $[\mathcal{K}]$ is a symmetric and positive-definite matrix and can then be written as $[\mathcal{K}]=\left[L_{K}\right]^{T}\left[L_{K}\right]$. Consequently, the nonparametric probabilistic approach can be extended to the geometrically nonlinear context and the construction of random matrix $[\mathcal{K}]$ is straightforward and is written as

$$
[\mathcal{K}]=\left[L_{K}\right]^{T}\left[\mathbf{G}_{K}\left(\delta_{K}^{N L}\right)\right]\left[L_{K}\right] .
$$

Then random matrix $[\mathcal{K}]$ can be block-decomposed similarly to Eq (5) so that the random stiffness terms $\mathcal{K}_{\alpha \beta}^{(e)}, \widehat{\mathcal{K}}_{\alpha \beta \gamma}^{(2)}$, and $\mathcal{K}_{\alpha \beta \gamma \delta}^{(3)}$ can easily be extracted. Furthermore, the random quadratic term $\mathcal{K}_{\alpha \beta \gamma}^{(2)}$ is then reconstructed from $\widehat{\mathcal{K}}_{\alpha \beta \gamma}^{(2)}$ similarly to Eq.(8).

\subsubsection{Analyzing the potential difficulties of the implementation}

When analyzing the nonlinear geometric response with respect to uncertainties, a difficulty arises if we want to establish a comparison between situations for which uncertainties affect both nonlinear and linear terms or only linear terms. Models (M1) and (M2) can not be used in order to set a comparison. The main reason is that the size of random germ $\left[\mathbf{G}_{K}\left(\delta_{K}^{N L}\right)\right]$ is thoroughly different from the size of random germ $\left[\mathbf{G}_{K^{(e)}}\left(\delta_{K^{(e)}}\right)\right]$. Furthermore, there is not any established criterion concerning the calibration of the dispersion parameters $\delta_{K}^{N L}$ and $\delta_{K^{(e)}}$ with respect to another one. In order to introduce some coherence when dealing with such a problem, having a common random germ for both situations is 
logically possible. Model (M2) has to also be used even if uncertainties only affect the linear elastic stiffness term. This requires to only extract random block $\mathcal{K}_{\alpha \beta}^{(e)}$ from random matrix $[\mathcal{K}]$, keeping the nonlinear quadratic and cubic terms as deterministic terms for the nonlinear calculations. Nevertheless, this case has been investigated in [26] in the context of the post-buckling nonlinear dynamical analysis of an uncertain cylindrical shell. With such method, a strong nonlinear level of uncertainty $\delta_{K}^{N L}=0.45$ yielded an equivalent linear uncertainty level $\delta_{K}^{L}=0.025$ for the linear part of the structure, yielding a subsequent scale effect, preventing to distinguish any differences between the linear and nonlinear uncertainty cases. Moreover, with such method, considering uncertainties on nonlinear terms would involve with $M^{2}(M+1)(M+2) / 2 \ggg M(M+1) / 2$ independent random variables. This makes the feasibility of generating random matrix $\left[\mathbf{G}_{K}\left(\delta_{K}^{N L}\right)\right]$ difficult as $M$ increases.

Consequently, the following section proposes an improvement of the methodology, allowing both models to be coherently considered.

\subsubsection{Improvement of the nonparametric modeling of uncertainties by using a second reduction}

The main idea is to use another factorization of matrix $[\mathcal{K}]$, which involves a rectangular matrix of dimension $(N \times P)$ with $N \lll P$. This is achieved by performing a reduction of matrix $[\mathcal{K}]$. Let us then consider the eigenvalue problem

$$
[\mathcal{K}] \boldsymbol{\psi}_{\alpha}=\lambda_{\alpha} \boldsymbol{\psi}_{\alpha}
$$

Matrix $[\mathcal{K}]$ can then be approximated by the $(P \times P)$ matrix $[\widetilde{\mathcal{K}}]$ such that

$$
[\widetilde{\mathcal{K}}]=\left[\widetilde{L}_{K}\right]^{T}\left[\widetilde{L}_{K}\right],
$$

in which $\left[\widetilde{L}_{K}\right]$ is the full $(N \times P)$ matrix defined by

$$
\left[\widetilde{L}_{K}\right]=\left[\Lambda^{N}\right]^{\frac{1}{2}}\left[\Psi^{N}\right]^{T},
$$

where $\left[\Lambda^{N}\right]$ is the $(N \times N)$ diagonal matrix such that $\left[\Lambda^{N}\right]_{\alpha \alpha}=\lambda_{\alpha}$, where $\lambda_{1} \geq \lambda_{2} \geq \cdots \geq \lambda_{N}$ and where the columns of the $(P \times N)$ matrix $\left[\Psi^{N}\right]$ are the eigenvector $\boldsymbol{\psi}_{\alpha}, \alpha \in\{1, \ldots, N\}$ related to eigenvalues $\lambda_{\alpha}$. In order to estimate the value of $N$, the error function $N \mapsto \operatorname{Conv}_{\mathrm{K}}(N)$ is defined such that

$$
\operatorname{Conv}_{\mathrm{K}}(N)=\sqrt{\frac{\|[\tilde{\mathcal{K}}]-\left.[\mathcal{K}]\right|_{F} ^{2}}{\|\left.[\mathcal{K}]\right|_{F} ^{2}}},
$$

in which $\|[A]\|_{F}$ is the Frobenius norm of matrix $[A] . N$ is chosen such that $\operatorname{Conv}_{\mathrm{K}}(N) \leq \varepsilon$ for a given relative error $\varepsilon$. Random matrix $[\mathcal{K}]$ is then replaced by the random matrix $[\widetilde{\mathcal{K}}]$ such that

$$
[\widetilde{\mathcal{K}}]=\left[\widetilde{L}_{K}\right]^{T}\left[\widetilde{\mathbf{G}}_{K}\left(\delta_{K}^{N L}\right)\right]\left[\widetilde{L}_{K}\right]+([\widetilde{\mathcal{K}}]-[\mathcal{K}])
$$

in which $\left[\widetilde{\mathbf{G}}_{K}\left(\delta_{K}^{N L}\right)\right]$ is a $(N \times N)$ random matrix with values in the set of the symmetric positive-definite matrices. Note that Eq. (14) ensures that stiffness operator $[\widetilde{\mathcal{K}}]$ is almost surely positive definite.

Concerning the computational savings related to this proposed method, it can be quantified as follows: using the usual method described in Section 2.2.2, the computational costs are due to

i. the Cholesky factorization of positive definite matrix $[\mathcal{K}]$, which is required once and yielding the $(P \times P)$ upper triangular matrix $\left[L_{K}\right]$.

ii. the construction of random matrix $\left[\mathbf{G}\left(\delta_{K}^{N L}\right)\right]=\left[\mathbf{L}_{G}\right]^{T}\left[\mathbf{L}_{G}\right]$, for which $\left[\mathbf{L}_{G}\right]$ is an upper random triangular matrix. Using the corresponding algebraic representation combined with $n_{s}$ realizations for the Monte Carlo numerical simulation, it requires the computation of $n_{s} P(P+1)$ independent random variables and the matrix multiplication requires about $\frac{2}{3} n_{s} P^{3}$ basic operations.

\section{Volume XX, Number XX, 2016}


iii. the construction of random matrix $[\mathcal{K}]$ according to Eq. (9) which requires about $2 n_{s} P^{3}$ basic operations.

Concerning the proposed method, the computational costs are due to

i. the numerical resolution of the eigenvalue problem of Eq.(10) and the construction of the full $(N \times P)$ matrix $\left[\widetilde{L}_{K}\right]$ according to Eq.(11), which is required once.

ii. the construction of random matrix $\left.\widetilde{[} \mathbf{G}\left(\delta_{K}^{N L}\right)\right]$, which requires the computation of $n_{s} N(N+1)$ independent random variables and about $\frac{2}{3} n_{s} N^{3}$ basic operations.

iii. the construction of random matrix $[\widetilde{\mathcal{K}}]$ according to Eq. (14) which requires about $4 n_{s} N^{2} P$ basic operations.

Introducing $r=\frac{P}{N}$, and neglecting the time costs induced by deterministic step (1) for both methods, it can be seen that the numerical gains concerning either the generation of the random variables and the generation of random matrices by matrix multiplication are approximatively $r^{2}$. It should be noted that the method is efficient with the condition $M \leq N \lll P$.

It should be noted that such methodology is particularly adapted for structures which are both sensitive to uncertainties and for which nonlinear geometrical effects can no longer be neglected. In this case, a stochastic nonlinear reduced-order model is needed in order to get an accurate representation of the solution. For instance, this is encountered for post-buckling situations which undergo large displacements and whose mechanical behavior is sensitive to any perturbation of the system with the inherent possibility of following several branches yielding different mechanical behaviors. This is also encountered in the context of mistuning turbomachinery, whose dynamical response is critically sensitive to any type of uncertainties. Investigating the case of nonlinear geometrical effects is equivalent to consider flutter situations yielding small damping and consequently large dynamical displacements. These two numerical situations are illustrated in the next Section.

This work is a part of a whole methodology developed through references [20, 25-27] where both uncertainties and nonlinear geometrical effects are simultaneously taken into account through the construction of an adapted stochastic nonlinear reduced order model. The second reduction proposed in this research, not only allows a computational gain as discussed above to be obtained but also allows several dynamical behaviours to be coherently compared. More particularly, uncertainties can affect only the linear stiffness or only the nonlinear stiffness terms as well as both. For these three situations, the random germs are identical. Matrix $[\widetilde{\mathcal{K}}]$ can be block decomposed similarly to Eq. (5). In the case for which uncertainties do only affect the linear stiffness terms, only the random elastic stiffness part is extracted from matrix $[\widetilde{\mathcal{K}}]$, using the nonlinear deterministic terms issued from the direct construction of the mean nonlinear reduced-order model. When considering uncertainties issued from the geometrical nonlinearities, random quadratic and cubic terms are extracted from matrix $[\widetilde{\mathcal{K}}]$ as explained in Section 2.2.2 for the model M2. The whole matrix is similarly used when considering uncertainties on both linear and nonlinear stiffness terms. The comparison between these three situations, for which the same random germ is used, is then adequate.

\section{NUMERICAL APPLICATIONS}

The proposed methodology is applied for two specific numerical cases with an industrial interest. The first one concerns the nonlinear static post-buckling analysis of a cylindrical shell and the second one is related to the nonlinear mistuning dynamical analysis of an industrial centrifugal compressor. 


\subsection{Uncertainty quantification for the static post-buckling of a cylindrical shell}

In this numerical application, the static post-buckling of an uncertain cylindrical shell is considered. In such case, the stochastic nonlinear reduced-order model is described from Eqs.(1) to (3) by the following equations:

$$
\begin{gathered}
\mathbf{U}=[\Phi] \mathbf{Q}, \\
{\left[\mathcal{K}^{(e)}\right] \mathbf{Q}+\mathcal{F}^{\mathrm{NL}}(\mathbf{Q})=\mathcal{F} .}
\end{gathered}
$$

\subsubsection{Description of the numerical model}

The structure under consideration is a cylindrical shell that is described in a global cartesian coordinate system $\left(O, \mathbf{e}_{1}, \mathbf{e}_{1}, \mathbf{e}_{3}\right)$, where $O$ is the center of the ring basis and where the cylinder axis is defined along $\mathbf{e}_{3}$. Its geometry is characterized by the mean radius $r_{m}=0.125 \mathrm{~m}$, thickness $e=270 \times 10^{-6} \mathrm{~m}$, and height $h=0.144 \mathrm{~m}$. The bottom of the cylindrical shell is clamped to its basis. The top of the cylindrical shell is rigid with three translation dof. Concerning the excitation, a constant traction load $F^{t}=8500 \mathrm{~N}$ is applied to delay the onset of the post-buckling. A controlled static shear point load $F^{\text {sh }}(s)$ with magnitude of $10,000 s$ measured in $N$, where $s$ is the increasing load dimensionless factor such that $s \in \mathcal{S}=[0,1.7]$, is then applied at the top of the shell along the direction $\mathbf{e}_{2}$. The material is a linear isotropic homogeneous elastic material in the range of stresses considered for which the Young modulus $E=1.8 \times 10^{11} \mathrm{Nm}^{-2}$ and the Poisson ratio $v=0.3$. Experimental results issued from this test case can be found in [37, 38]. The finite element model of the structure is constituted of a regular mesh of $\left(n_{r}-1\right) \times n_{\theta} \times\left(n_{3}-1\right)=1 \times 7,500 \times 9,599=712,500$ tridimensional solid finite elements with 8 nodes, in which $n_{r}, n_{\theta}$ and $n_{3}$ are the number of nodes along the radial, orthoradial and $\mathbf{e}_{3}$ directions. The numerical model has $4,230,003$ dof's. The chosen observation $u_{o b s}(s)$ is defined at the rigid body motion at $x_{3}=h$ along the shear loading direction. It should be noted that the structure is lightly numerically pre-deformed according to the first Euler buckling mode, represented by figure 1 with a maximum amplitude of $e$. More details concerning the construction of the numerical model can be found in [26].

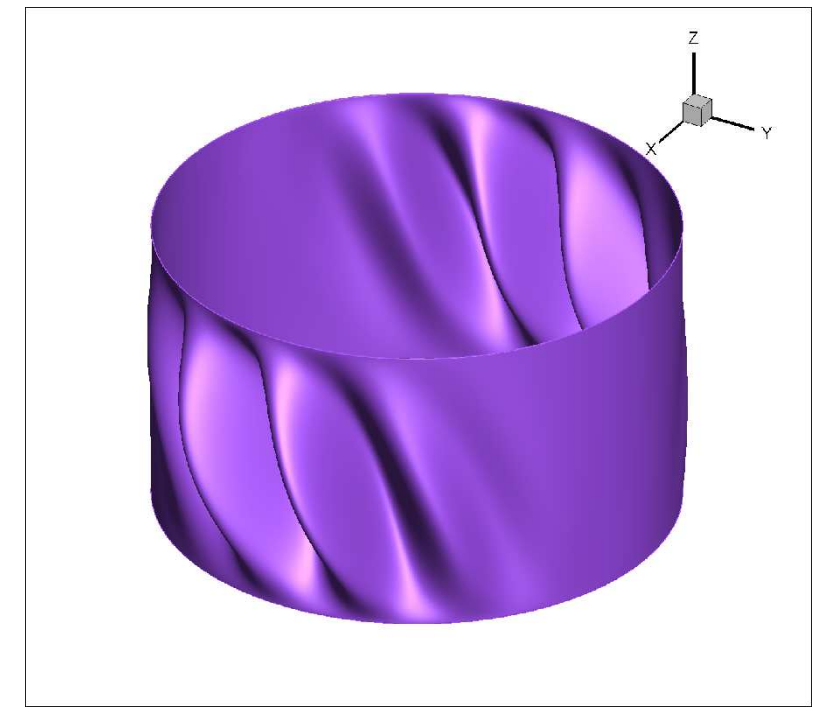

FIG. 1: First Euler buckling mode of the cylindrical shell. 


\subsubsection{Convergence analysis}

The mean nonlinear reduced-order model is constructed from the nonlinear static response from the Proper Orthogonal Decomposition method [28, 29, 31, 39] with $M=27$ vector basis in order to accurately represent the nonlinear static solution [26]. Consequently, the operator $[\mathcal{K}]$ defined by Eq.(5) is a square matrix of order $P=756$. Using the second reduction method described in Section 2.2.2, let $[\widetilde{\mathcal{K}}]$ be the approximation of matrix $[\mathcal{K}]$ obtained by keeping the $N$ greatest eigenvalues. Figure 2 displays the graph of function $N \mapsto \operatorname{Conv}_{\mathrm{K}}(N)$ defined by Eq. (13). It can be shown that the convergence is fastly obtained. From now on, we choose $N=425$ that is a value smaller than $P=756$.

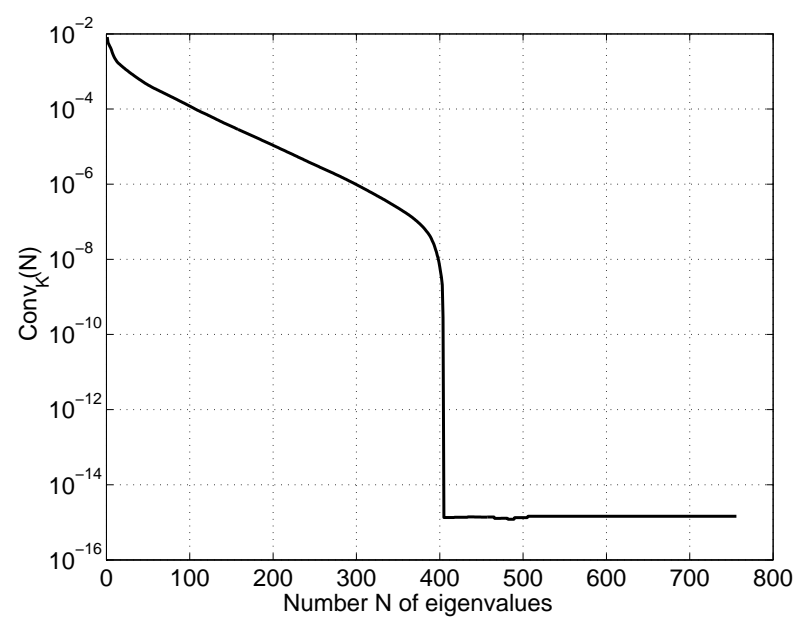

FIG. 2: Convergence analysis concerning the second reduction method: graph of function $N \mapsto$ $\operatorname{Conv}_{\mathrm{K}}(N)$.

With the presence of random uncertainties, the nonlinear displacement observation $U_{o b s}(s)$, obtained for a given increment load $s$ is a random variable. For the present case, the uncertainty level is described by dispersion parameter $\delta=0.1$ for the random operator $[\widetilde{\mathcal{K}}]$ defined by Eq. (14). The linear elastic stiffness is uncertain, whereas the nonlinear terms remain deterministic. The convergence of the nonlinear stochastic response with respect to the second reduction method is then analyzed with respect to the number $n_{s}$ of realizations required by the Monte Carlo numerical simulation and with respect to the size $N$ of the second-order reduction method by introducing the following quantity

$$
\operatorname{Conv}_{2}\left(N, n_{s}\right)=\sqrt{\frac{1}{n_{s}} \sum_{j=1}^{n_{s}}\left(\int_{\mathcal{S}}\left(\widetilde{U}_{o b s}^{N}\left(s, \theta_{j}\right)\right)^{2} d s\right)},
$$

in which $\widetilde{U}_{o b s}^{N}\left(s, \theta_{j}\right)$ is the realization $\theta_{j}$ of the approximation of random observation $U_{o b s}(s)$ obtained with the second reduction method of size $N$. Figures 3 and 4 display the graphs $N \mapsto \operatorname{Conv}_{2}(N, 3,000)$ and $n_{s} \mapsto \operatorname{Conv}_{2}\left(425, n_{s}\right)$. It can be concluded that a good approximation is obtained with $N=425$ and $n_{s}=2,000$.

\subsubsection{Stochastic analysis of the nonlinear static post-buckling response of the cylindrical shell.}

In this Section, the stochastic nonlinear response is analyzed with respect to the load increment $s$. Two distinct uncertain branches related to the nonlinear behavior are observed. There exist some realizations for which the nonlinear response behavior is similar to the nonlinear response of the mean model. In 


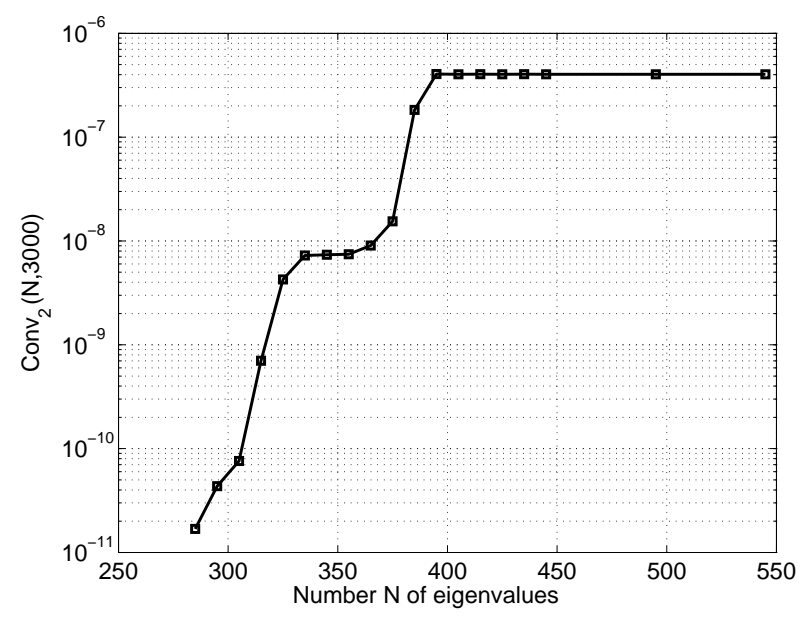

FIG. 3: Stochastic convergence analysis of the random response: graph $N \mapsto \operatorname{Conv}_{2}(N, 3,000)$.

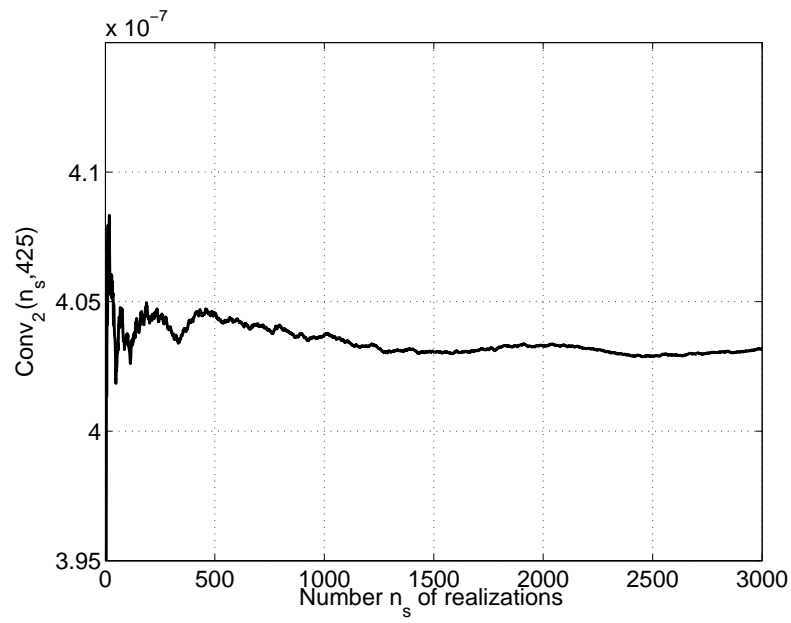

FIG. 4: Stochastic convergence analysis of the random response: graph $n_{s} \mapsto \operatorname{Conv}_{2}\left(425, n_{s}\right)$.

such case, it is observed that the nonlinear stochastic response is quasilinear and particularly robust with respect to uncertainties when $s<0.6$. The effects of uncertainties then increase with the nonlinear effects. When the load increment $s$ belongs to $[0.6,1]$, we observe a sudden softening, for which the confidence region quickly broadens and which corresponds to the post-buckling range. Finally, for $s>1$, an elastic stiffening behavior appears, which is more robust to uncertainties and whose robustness remains uniform with the load increment $s$. Secondly, another branch of nonlinear behavior is observed. In this case, we again observe for $s<0.6$ a quasi-deterministic linear elastic response. For $s>0.6$, a slight progressive softening of the response combined with a regular increasing sensitivity with respect to uncertainties is observed. Consequently, each uncertain branch of nonlinear response is characterized by the random observations $U_{o b s}^{1}(s, \delta=0.1)$ and $U_{o b s}^{2}(s, \delta=0.1)$, whose confidence regions are represented by the graphs in figures 5 and 6 .

Figures 7 and 8 represent the static post-buckling deformation of the cylindrical shell for a load increment $s=1.6$. Both structures buckle according to the first Euler buckling mode. Nevertheless, the two observed branches differ by its softening evolution in the post-buckling range. 


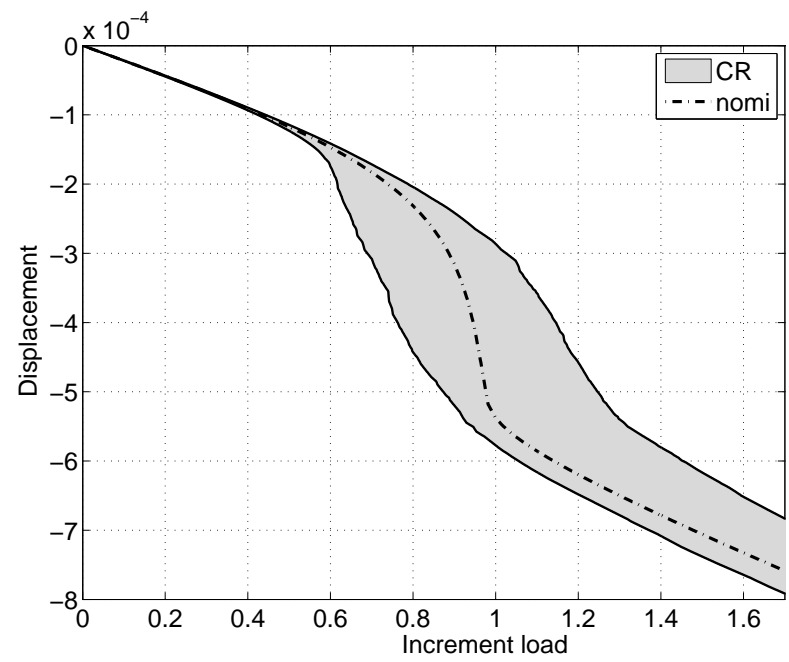

FIG. 5: Graph of the mean nonlinear response $s \mapsto u_{o b s}(s)$, confidence region of random observation $s \mapsto U_{o b s}^{1}(s, \delta=0.1)$.

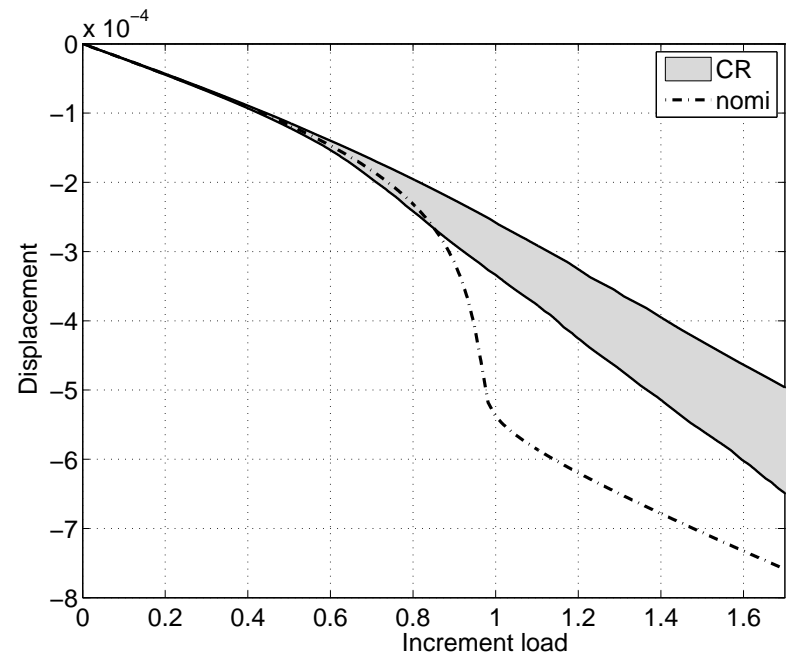

FIG. 6: Graph of the mean nonlinear response $s \mapsto u_{o b s}(s)$, confidence region of random observation $s \mapsto U_{o b s}^{2}(s, \delta=0.1)$.

Finally, it should be noted that when uncertainties affect simultaneously both linear and nonlinear stiffness terms, only one post-buckling behavior similar to the one shown in figure 5 is observed.

\subsection{Uncertainty quantification for a nonlinear mistuned industrial centrifugal compres- sor}

In this numerical application, the nonlinear dynamics of a mistuned rotating structure with geometrical nonlinear effects are considered. The mistuning phenomenon, which destroys the natural cyclic symmetry of the bladed disk, is induced by manufacturing tolerances and variabilities of the mechanical properties, which create small discrepancies from one blade to another one. Its random character leads 


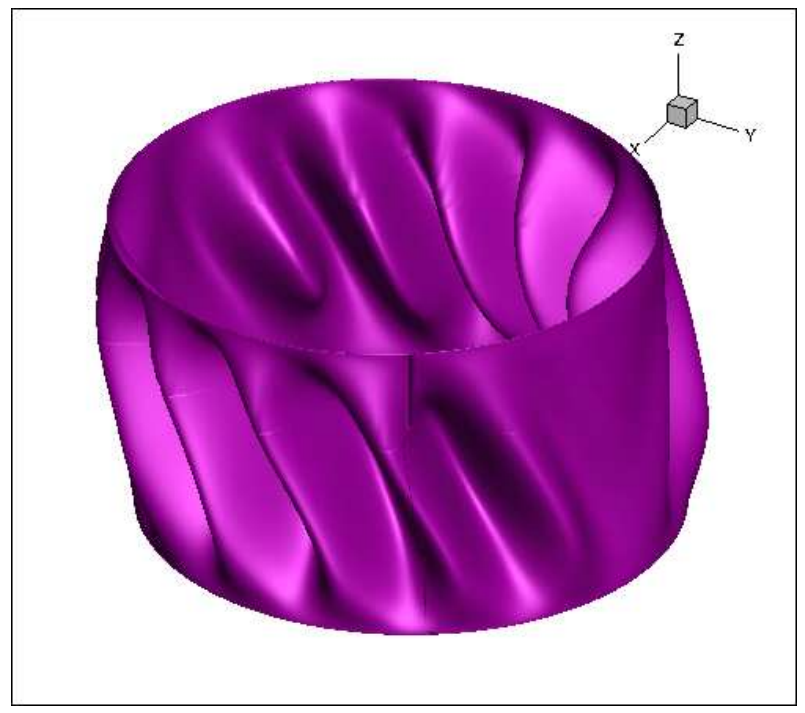

FIG. 7: Static post-buckling of a realization $\theta$ of random observation $U_{o b s}^{1}(s=1.6, \delta=0.1)$.

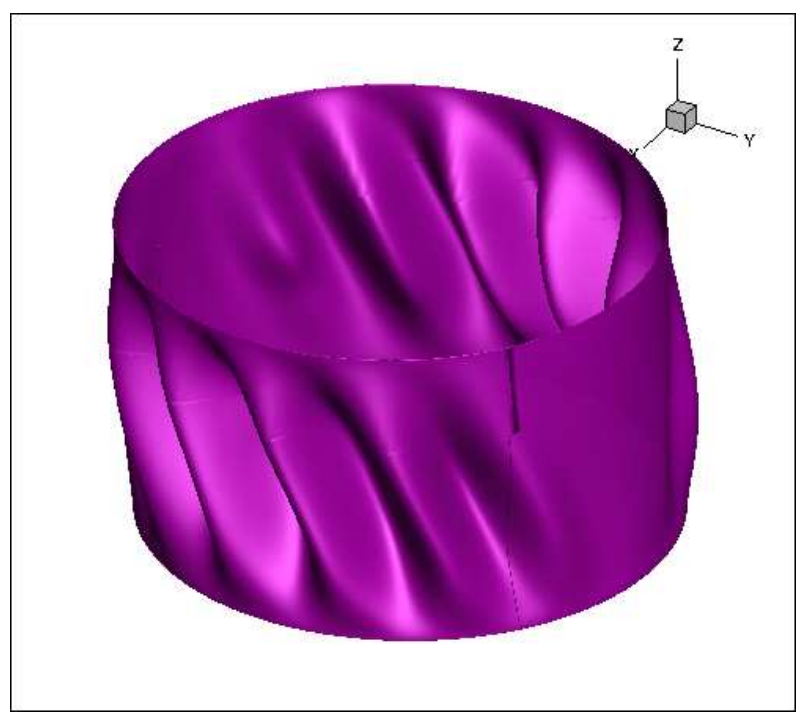

FIG. 8: Static post-buckling of a realization $\theta$ of random observation $U_{\text {obs }}^{2}(s=1.6, \delta=0.1)$.

to uncertainties and it is well known that the dynamical response is very sensitive to such mistuning [40].

In such a context, the dynamics of this structure is described by the following stochastic nonlinear reduced-order model deduced from Eqs. (1) to (3):

$$
\begin{gathered}
\mathbf{U}=[\Phi] \mathbf{Q} \\
{[\mathcal{M}] \ddot{\mathbf{Q}}+([\mathcal{D}]+[\mathcal{C}(\Omega)]) \dot{\mathbf{Q}}+\left(\left[\mathcal{K}^{(e)}\right]+\left[\mathcal{K}^{(c)}(\Omega)\right]\right) \mathbf{Q}+\mathcal{F}^{\mathrm{NL}}(\mathbf{Q})=\mathcal{F} .}
\end{gathered}
$$




\subsubsection{Description of the numerical model}

The structure under consideration is an industrial centrifugal compressor belonging to the class of integrated bladed disks. Due to the industrial proprietary reasons, the number $M$ of blades characterizing the order of the cyclic symmetry of the structure cannot be given. The finite element model of the structure is constructed with solid finite elements and is constituted of about 2,000,000 dof's. The structure is in rotation around its revolution axis with a constant velocity $\Omega=30,750 \mathrm{rpm}$. Since the dynamic analysis is carried out in the rotating frame of the structure, the rigid body motion due to the rotation of the structure corresponds to a fixed boundary condition at the inner radius of the structure. The bladed disk is made up of a homogeneous isotropic material. A modal damping is added in the computational model of the bladed disk.

\subsubsection{Convergence analysis}

The mean nonlinear reduced-order model is constructed by using $M=60$ elastic modes of the linear tuned structure with cyclic symmetry, as shown in [27]. Consequently, $[\mathcal{K}]$ is a square matrix of order $P=3,660$. Figure 9 displays the graph $N \mapsto \operatorname{Conv}_{\mathrm{K}}(N)$ for which it can be shown that the convergence is fastly obtained. From now on, we choose $N=400$.

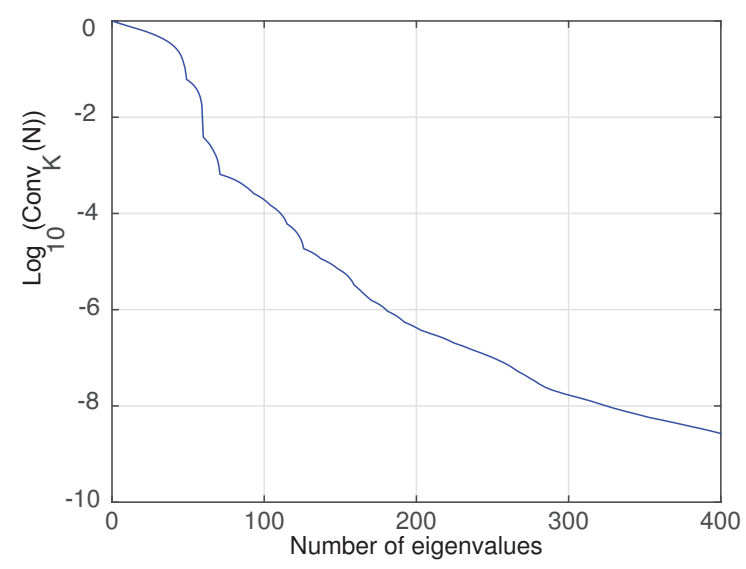

FIG. 9: Convergence analysis concerning the second reduction method: graph of function $N \mapsto$ $\log _{10}\left(\operatorname{Conv}_{\mathrm{K}}(N)\right)$.

Let $v_{0}$ be the first eigenfrequency of the structure. All the quantities defined in the frequency domain are expressed with respect to $v / v_{0}$ in which $v$ is the physical frequency. The external load is a 5 th engineorder excitation expressed in the time domain for which the corresponding dimensionless frequency excitation band is $\mathbb{B}_{\text {exc }}=[1.78,2.34]$ with an intensity load taken as $2.5 \mathrm{~N}$. It is located at the tip of each blade [36]. The random observation $W(2 \pi v)$ corresponds to the random displacement located at the tip of the most responding blade.

As shown in [27], it is seen that the geometric nonlinearities propagate the random response of the blade outside $\mathbb{B}_{\text {exc }}$. More particularly, it is seen that the resonance located outside $\mathbb{B}_{\text {exc }}$ has not only a non-negligible contribution but also is particularly sensitive to uncertainties. Such complex dynamical behavior yields to introduce the two random observations $W_{\infty}^{1}$ and $W_{\infty}^{2}$ such that

$$
W_{\infty}^{1}=\max _{v \in \mathbb{B}_{\text {exc }}} W(2 \pi v) \quad W_{\infty}^{2}=\max _{v \in \mathbb{B}_{\text {sub }}} W(2 \pi v)
$$


in which $\mathbb{B}_{\text {sub }}=[1 ; 1.6]$ is a part of the frequency band of observation located below $\mathbb{B}_{\text {exc }}$. The convergence of the random solution is then analyzed with respect to the number $n_{s}$ of realizations required by the Monte Carlo numerical simulation and the size $N$ of the second-order reduction method by introducing the following quantity

$$
\operatorname{Conv}_{\infty}^{i}\left(N, n_{s}\right)=\sqrt{\frac{1}{n_{s}} \sum_{j=1}^{n_{s}}\left(\widetilde{W}_{\infty}^{N, i}\left(\theta_{j}\right)\right)^{2}}
$$

in which $\widetilde{W}_{\infty}^{N, i}\left(\theta_{j}\right)$ is the realization $\theta_{j}$ of the approximation of random observation $W_{\infty}^{i}$ obtained with the second reduction method of size $N$. Figure 10 and 11 display the graphs $N \mapsto \operatorname{Conv}_{\infty}^{i}(N, 1,000)$ and $n_{s} \mapsto \operatorname{Conv}_{\infty}^{i}\left(200, n_{s}\right)$. It can be concluded that a good approximation is obtained with $N=200$ and $n_{s}=600$.

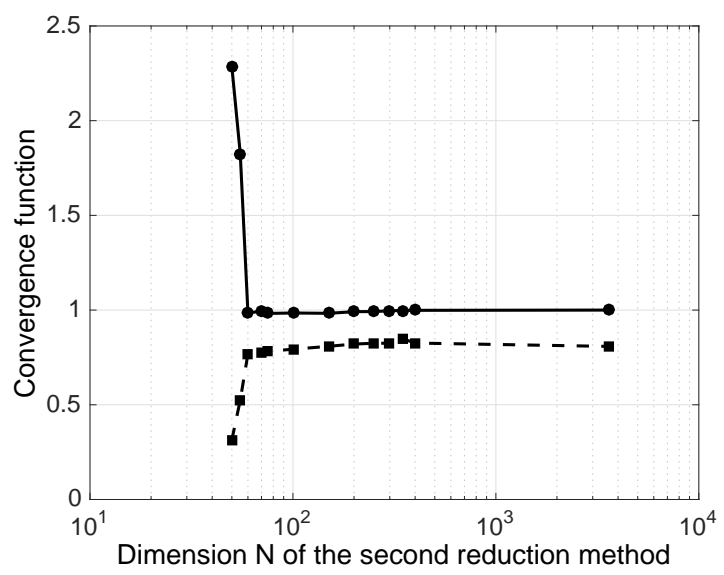

FIG. 10: Stochastic convergence analysis of the random response: graph $N \mapsto \operatorname{Conv}_{\infty}^{i}(N, 1,000)(1)$ main resonance (solid line) (2) secondary resonance (dotted line).

\subsection{Quantification of the nonlinear mistuning with respect to the linear mistuning}

Until now, it has been assumed in the industrial application [27] that the mistuning only affects the linear operators. In this case, that means that linear mistuning in presence of deterministic geometric nonlinearities is considered. Concerning the operators involved by the linear elastic constitutive equations, only the linear elastic stiffness operator is uncertain, the quadratic and cubic terms remaining deterministic. From now on, we are interested in considering the case for which uncertainties affect all the operators due to the elastic constitutive equations with nonlinear geometrical effects. This will be called nonlinear mistuning, that is to say both the linear and nonlinear stiffness operators are affected by uncertainties.

Figures 12 and 13 compare the random observation for the cases of nonlinear and linear mistuning respectively. There are slight differences between the two cases. It can be observed that the presence of uncertainties on the nonlinear part of the stiffness operator yields narrower confidence region in the sub-frequency range. Note that this conclusion does not hold for the static post-buckling application presented in Section 3.1 and that such a conclusion cannot a priori be used for any nonlinear dynamical system. Since there are slight differences between Figures 12 and 13, the case corresponding to pure nonlinear mistuning is investigated. By comparing figures 12 and 14, it can be seen that the pure nonlinear mistuning yields a quasi-deterministic response in the frequency band of excitation whereas the 


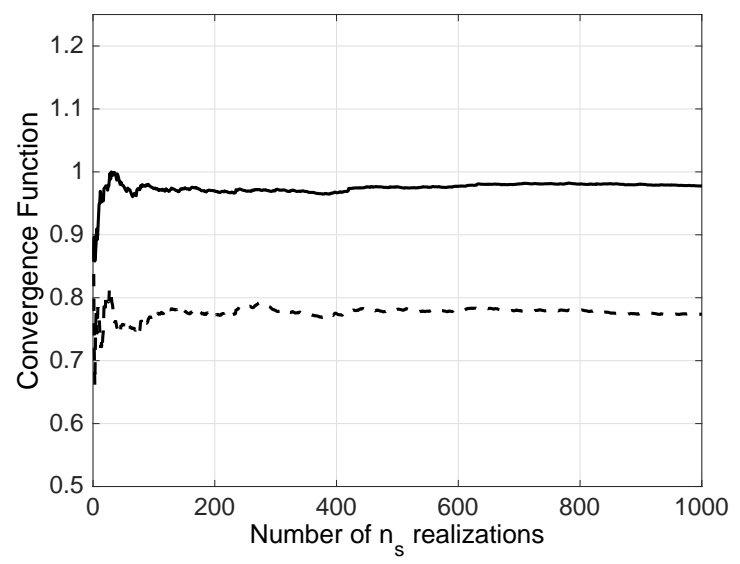

FIG. 11: Stochastic convergence analysis of the random response: graph $n_{s} \mapsto \operatorname{Conv}_{\infty}^{i}\left(200, n_{s}\right)$ (1) main resonance (solid line) (2) secondary resonance (dotted line).

effects of uncertainty propagation induced by either nonlinear or linear mistuning remain similar outside the excitation frequency band. Finally, note that the numerical computations have been made with the same convergence numerical parameters $N=200$ and $n_{s}=600$ as found through the convergence analysis performed for the linear mistuned case (in presence of determinstic geometrical nonlinearities).

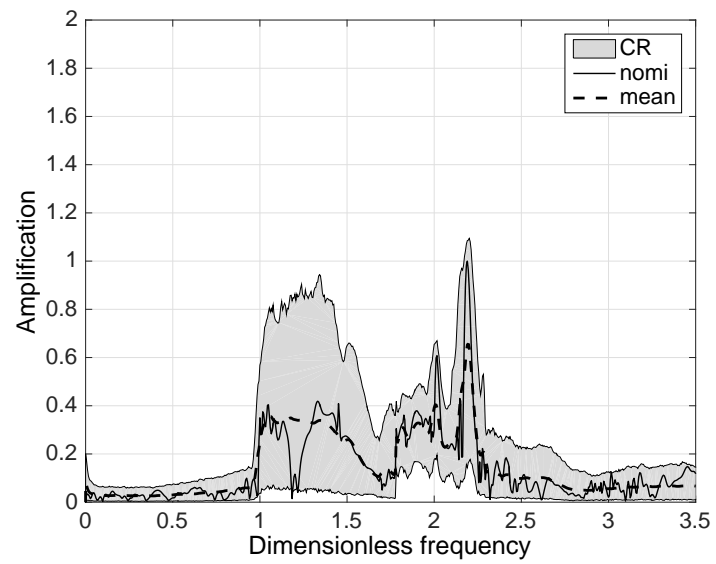

FIG. 12: Stochastic analysis for the nonlinear mistuning: random observation $W(2 \pi v)(1)$ mean model (thick line), mean of the stochastic model (thin dashed line), and confidence region(gray region). 


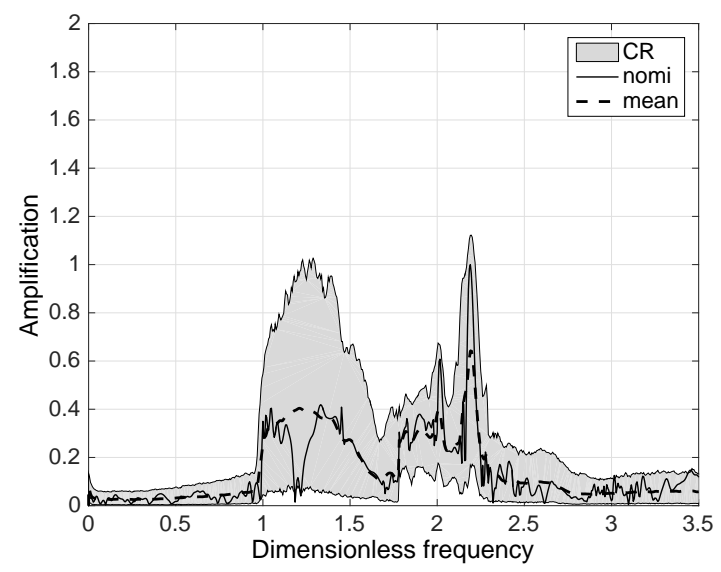

FIG. 13: Stochastic analysis for the linear mistuning: random observation $W(2 \pi v)(1)$ mean model (thick line), mean of the stochastic model (thin dashed line), and confidence region(gray region).

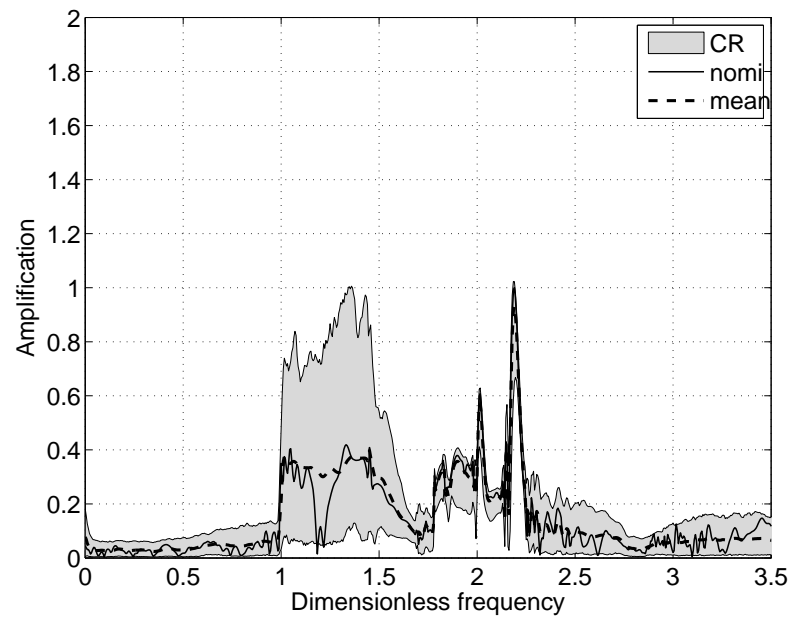

FIG. 14: Stochastic analysis for the pure nonlinear mistuning: random observation $W(2 \pi v)(1)$ mean model (thick line), mean of the stochastic model (thin dashed line), and confidence region(gray region).

\section{CONCLUSIONS}

In the context of structural dynamics with geometric nonlinearities, a method has been presented for quantifying the propagation of uncertainties whether the uncertainties are applied to the linear operators or is applied to both the linear and nonlinear operators. The method is adapted to the case for which the dimension of the reduced-order model is high. It has been applied to the context of the nonlinear static post-buckling of a thin cylinder and to the context of both linear and nonlinear mistuning of an industrial integrated bladed-disk. The results are quite different and depend on the nature of the application. The post-buckling behavior of the cylinder is really sensitive to the type of uncertainty modelling. It let to observe two distinct types of post-buckling responses. In opposite, the mistuned bladed disk yields a similar uncertain nonlinear dynamical response when considering linear or nonlinear mistuning outside 
the excitation frequency band. It can also be seen that a pure nonlinear mistuning yields a particular robust response in the excitation frequency band.

\section{ACKNOWLEDGMENTS}

This work was supported by the DGA (French defense procurement agency) in the context of the TURBODYNA project (project number ANR-13-ASTR-0008-01) related to the ANR ASTRID research program (specific support scheme for research works and innovation defense). SAFRAN Helicopter Engines is also acknowledged for giving the finite element model to publish this work.

\section{REFERENCES}

1. Laxalde, D., Thouverez, F., Sinou, J.-J., and Lombard, J.-P., Qualitative analysis of forced response of blisks with friction ring dampers, European Journal of Mechanics, A/Solids, 26(4):676-687, 2007.

2. Petrov, E.-P., Analysis of sensitivity and robustness of forced response for nonlinear dynamic structures, Mechanical Systems and Signal Processing, 23(1):68-86, 2009.

3. Petrov, E.-P., Analysis of flutter-induced limit cycle oscillations in gas-turbine structures with friction, gap, and other nonlinear contact interfaces, Journal of Turbomachinery, 134(6):061018-1-13, 2012.

4. Martins, A., Simoes, L., and Negrao, J., Optimization of cable forces on concrete cable-stayed bridges including geometrical nonlinearities, Computers and Structures, 155:18-27, 2015.

5. Sun, X. and Jing, X., Multi-direction vibration isolation with quasi-zero stiffness by employing geometrical nonlinearity, Mechanical Systems and Signal Processing, 62-63:149-163, 2015.

6. Pai, P. and Nayfeh, A., A Fully Nonlinear-Theory of Curved And Twisted Composite Rotor Blades Accounting For Warpings and 3-Dimensional Stress Effects, International Journal of Solids and Structures, 31(9):1309-1340, MAY 1994.

7. Tang, D.-M. and Dowell, E., Nonlinear response of a non-rotating rotor blade to a periodic gust, Journal of Fluids and Structures, 10(7):721-742, OCT 1996.

8. Grolet, A. and Thouverez, F., Free and forced vibration analysis of a nonlinear system with cyclic symmetry: Application to a simplified model, Journal of Sound and Vibration, 331(12):2911-2928, 2012.

9. Elishakoff, I., Uncertain buckling: its past, present and future, International Journal of Solids and Structures, 37(46-47):6869-6889, 2000.

10. Schenk, C. and Schueller, G., Buckling analysis of cylindrical shells with random geometric imperfections, International Journal of Nonlinear Mechanics, 38(7):1119-1132, 2003.

11. Papadopoulos, V., Stefanou, G., and Papadraakis, M., Buckling analysis of imperfect shells with stochastic non-gaussian materials and thickness properties, International Journal of Solids and Structures, 46(14-15):2800-2808, 2009.

12. Hodges, D.-H., Shang, X.-Y., and Cesnik, C., Finite element solution of nonlinear intrinsic equations for curved composite beams, Journal of the American Helicopter Society, 41(4):313-321, OCT 1996.

13. Huang, H. and Han, Q., Research on nonlinear postbuckling of functionally graded cylindrical shells under radial loads, Composite Structures, 92(6):1352-1357, MAY 2010.

14. Schillinger, D., Papadopoulos, V., Bischoff, M., and Papadrakakis, M., Buckling analysis of imperfect i-sections beam-columns with stochastic shell finite elements, Computational Mechanics, 46(3):495-510, 2010. 
15. Elishakoff, I., Kriegesmann, B., and Rolfes, R., Optimization and antioptimization of buckling load for composite cylindrical shells under uncertainties, AIAA Journal, 50(7):1513-1524, 2012.

16. Muryavov, A. and Rizzi, S., Determination of nonlinear stiffness with application to random vibration of geometrically nonlinear structures, Computers and Structures, 81:1513-1523, 2003.

17. Grepl, M., Maday, Y., Nguyen, N., and Patera, A., Efficient reduced-basis treatment of nonaffine and nonlinear partial differential equations., ESAIM: Mathematical Modelling and Numerical Analysis, 41(3):575-605, 2007.

18. Nguyen, N. and Peraire, J., An efficient reduced-order modeling approach for non-linear parametrized partial differential equations., International Journal for Numerical Methods in Engineering, 76(1):27-55, 2008.

19. Amsallem, D., Zahr, M., and Farhat, C., Nonlinear model order reduction based on local reducedorder bases., International Journal for Numerical Methods in Engineering, 92(10):891-916, 2012.

20. Capiez-Lernout, E., Soize, C., and Mignolet, M.-P., Computational stochastic statics of an uncertain curved structure with geometrical nonlinearity in three-dimensional elasticity, Computational Mechanics, 49(1):87-97, 2012.

21. Mignolet, M.-P., Przekop, A., Rizzi, S., and Spottswood, M., review of indirect/non-intrusive reduced-order modeling of nonlinear geometric structures, Journal of Sound and Vibration, 332(10):2437-2460, 2013.

22. Gaonkar, A. and Kulkami, S., Model order reduction for dynamic simulation of beams with forcing and geometric nonlinearities, Finite Elements in Analysis and Design, 76:50-62, 2013.

23. Touze, C., Vidrascu, M., and Chapelle, D., Direct finite element computation of non-linear modal coupling coefficients for reduced-order shell models, Computational Mechanics, 54(2):567-580, 2014.

24. Farhat, C., Avery, P., Chapman, T., and Cortial, J., Dimensional reduction of nonlinear finite element dynamic models with finite rotations and energy-based mesh sampling and weighting for computational efficiency., International Journal for Numerical Methods in Engineering, 98(9):625662, 2014.

25. Mignolet, M.-P. and Soize, C., Stochastic reduced-order models for uncertain geometrically nonlinear dynamical systems, Computer Methods in Applied Mechanics and Engineering, 197:39513963, 2008.

26. Capiez-Lernout, E., Soize, C., and Mignolet, M.-P., Post-buckling nonlinear static and dynamical analyses of uncertain cylindrical shells and experimental validation, Computer Methods in Applied Mechanics and Engineering, 271:210-230, 2014.

27. Capiez-Lernout, E., Soize, C., and Mbaye, M., Mistuning analysis and uncertainty quantification of an industrial bladed disk with geometrical nonlinearity, Journal of Sound and Vibration, 356(11):124-143, 2015.

28. Sirovich, L., Turbulence and the dynamics of coherent structures, Quarterly of Applied Mathematics, 45(3):561-571, OCT 1987.

29. Azeez, M. and Vakakis, A.-F., Proper orthogonal decomposition (POD) of a class of vibroimpact oscillations, Journal of Sound and Vibration, 240(5):859-889, 2001.

30. Kerschen, G., Golinval, J., Vakakis, A., and Bergman, L., The method of proper orthogonal decomposition for dynamical characterization and order reduction of mechanical systems: An overview., Nonlinear Dynamics, 41(1-3):147-169, 2005.

31. Sampaio, R. and Soize, C., Remarks on the efficiency of pod for model reduction in non-linear dynamics of continuous elastic systems, Journal of Sound and Vibration, 72(1):22-45, 2007. 
32. Soize, C., Medium frequency linear vibrations of anisotropic elastic structures., La recherche aérospatiale (english version), 5:65-87, 1982.

33. Soize, C., Stochastic Models of Uncertainties in Computational Mechanics, Lecture Notes in Engineering Mechanics 2, American Society of Civil Engineers (ASCE), 2012.

34. Crisfield, M., Non-linear finite element analysis of solids and structures, Vol. 1 : essentials, John Wiley and Sons, Chichester, 1997.

35. Soize, C., A nonparametric model of random uncertainties for reduced matrix models in structural dynamics, Probabilistic Engineering Mechanics, 15(3):277-294, 2000.

36. Capiez-Lernout, E., Soize, C., and Mbaye, M., Uncertainty quantification for an industrial mistuned bladed disk with geometrical nonlinearities, In paper GT2015-42471, Proceedings of the ASME Turbo Expo 2015: Turbine Technical Conference and Exposition GT2015, June 15-19, 2015, Montréal, Canada, 2015.

37. Michel, G., Combescure, A., and Jullien, J.-F., Finite element simulation of dynamic buckling of cylinders subjected to periodic shear, Thin-Walled Structures, 36:111-135, 2000.

38. Michel, G., Limam, A., and Jullien, J.-F., Buckling of cylindrical shells under static and dynamic shear loading, Engineering Structures, 22:535-543, 2000.

39. Chaturantabut, S. and Sorensen, D. C., A state space error estimate for pod-deim nonlinear model reduction, SIAM Journal on numerical analysis, 50(1):46-63, 2012.

40. Wei, S.-T. and Pierre, C., Localization phenomena in mistuned assemblies with cyclic symmetry part ii: Forced vibrations, ASME Journal of vibration acoustics stress and reliability in design, 110(4):439-449, 1988. 